\title{
Patient-specific exercise programs in the conservative management of the so-called idiopathic scoliosis
}

\section{T Karski}

Address: Department of Pediatric Orthopedics and Rehabilitation of Skubiszewski Medical University of Lublin; University Pediatric Hospital, Chodźki St. 2, 20-093 Lublin, Poland

Email: T Karski - tkarski@dsk.lublin.pl

from 4th International Conference on Conservative Management of Spinal Deformities

Boston, MA, USA. 13-16 May 2007

Published: 12 October 2007

Scoliosis 2007, 2(Suppl I):P5 doi:I0.II86/I748-7|6I-2-SI-P5

This abstract is available from: http://www.scoliosisjournal.com/content/2/SI/P5

(c) 2007 Karski; licensee BioMed Central Ltd.

\section{Objective}

To describe the application of a new classification system for early identification of patients that will benefit from specific exercise programs designed to prevent deformity development and progression

\section{Study design}

A group of 629 children, aged four to eight years, were identified in school scoliosis screening programs. Children judged to be at risk were assigned to three groups based on diagnostic protocols to identify asymmetry in posture and movement correlated with curvature patterns.

\section{Methods}

Exercise programs designed to improve flexion and stretching, and not strengthening, were used.

\section{Results}

Among treated patients, curvatures in seventy-one percent either improved or remained stable. Sixteen percent progressed significantly, with thirteen percent progressing to the point of requiring operative intervention.

\section{Conclusion}

Successful exercise-based rehabilitation requires early detection combined with programs designed for specific signs and symptoms in children at risk for progression. 